\title{
Incidencia de la anormalidad de la vértebra de transición, en la población mexicana de un hospital privado durante 3 años
}

\author{
Incidence of transitional vertebra abnormality in the Mexican \\ population of a private hospital during 3 years
}

\author{
Víctor Raúl Carbajal Saldaña, ${ }^{*}$ Georgina Cornelio Rodríguez, \\ José Luis Ríos Reina, ${ }^{\S}$ Carolina González Vergara ${ }^{\S}$
}

Citar como: Carbajal SVR, Cornelio RG, Ríos RJL, González VC. Incidencia de la anormalidad de la vértebra de transición, en la población mexicana de un hospital privado durante 3 años. Acta Med GA. 2022; 20 (1): 7-11. https://dx.doi.org/10.35366/103549

\section{Resumen}

La vértebra de transición es una anormalidad morfológica de la vértebra L5 o S1 donde alguna de las dos vértebras o ambas presenta un proceso displásico y asemeja a la otra vértebra, lo cual conlleva diversos problemas como escoliosis o alteración del equilibrio, entre otros. Esta patología muchas veces está infradiagnosticada debido a que suele ser un hallazgo azaroso en los pacientes. Objetivo: Determinar la incidencia de la anormalidad de la vértebra de transición por tomografía computada en la población mexicana durante tres años. Material y métodos: Estudio observacional, descriptivo, retrospectivo y transversal. Se incluirán todos los casos de vértebra de transición detectados en tres años en el Hospital Angeles Mocel. Resultados: De 860 pacientes estudiados con una edad media de $51.99 \pm 20.54$ años se encontró una incidencia de $17.4 \%(n=150)$ para vértebra de transición, donde 96 casos presentaron proceso de sacralización. La vértebra de transición de tipo IIA fue la más común y el tipo de pseudodisco tipo 4. Conclusión: La incidencia en la población mexicana presentada fue de $17.4 \%$, similar a lo reportado en otras latitudes, con características similares a lo señalado para la vértebra de transición.

Palabras clave: Vértebra de transición, radiología, imagen, tomografía computada.

\section{Abstract}

The transitional vertebra is a morphological abnormality of the L5 or S1 vertebra where one of the two vertebrae or both presents a dysplasic process and resembles the other vertebra, which leads to various problems such as scoliosis or alteration of balance among others. This pathology is often underdiagnosed due to the fact that it is usually a random finding in patients. Objective: To determine the incidence of abnormality of the transitional vertebra by computed tomography in the Mexican population for three years. Material and methods: Observational, descriptive, retrospective, and cross-sectional study. All cases of transition vertebra detected in three years at Hospital Angeles Mocel will be included. Results: Of 860 patients studied with a mean age of $51.99 \pm$ 20.54 years, an incidence of $17.4 \%(n=150)$ was found for transitional vertebra, where 96 cases presented a process of sacralization. The type IIA transitional vertebra was the most common and the type 4 pseudo-disc type. Conclusion: The incidence in the Mexican population presented was $17.4 \%$ similar to that reported in other latitudes, with characteristics similar to those indicated for the transitional vertebra.

Keywords: Transitional vertebra, radiology, imaging, computed tomography.

* Servicio de Radiología e Imagen. Facultad Mexicana de Medicina de la Universidad La Salle. Hospital Angeles Mocel/Metropolitano. México.

₹ Servicio de Radiología e Imagen. Hospital Angeles Roma/Pedregal. México.

§ Servicio de Radiología e Imagen. Hospital Angeles Mocel. México. $\quad$ Aceptado: 12-04-2021. 


\section{INTRODUCCIÓN}

La vértebra de transición es una anormalidad congénita de la región lumbosacra que incluye tanto la lumbarización como la sacralización, derivada de un defecto en la segmentación lumbosacra en el pedido de desarrollo fetal. ${ }^{1}$ Ésta se presenta en toda la población con una incidencia descrita en la actualidad de entre 4 a $30 \%$ variando su incidencia entre distintos países. ${ }^{2}$ Aunque los estudios más específicos o extendidos señalan una incidencia de entre $3.3-35.6 \% .^{3}$

Con diferentes variaciones en cuanto al rango o grado morfológico de diferenciación, que van en segmentos desde las vértebras $L 5$ con procesos transversales alargados ensanchados hasta la fusión completa con el sacro denominando a este proceso como lumbarización. Mientras que, si el segmento sacro $\mathrm{S} 1$ muestra procesos o diversos grados de similitud a las vértebras lumbares, como la formación de una articulación anómala a la configuración sacra con una imagen cuadrada en el plano sagital y un disco más pequeño de lo habitual, se denomina proceso sacralización. ${ }^{4}$

En cuanto a su etiología, es desconocida en su totalidad, aunque en la actualidad se cree que exista un componente genético envuelto en la incidencia de la vértebra de transición, considerando que los genes partícipes podrían ser Hox-10 y Hox-11, que intervienen en la segmentación axial del esqueleto durante la embriogénesis. ${ }^{5}$

Su diagnóstico e identificación se da meramente por estudios de imagen, teniendo como estudio inicial el uso de la radiografía; aunque la principal técnica de imagen útil para un correcto diagnóstico de la vértebra de transición se da mediante el uso de la tomografía computada gracias a su gran calidad de imagen. ${ }^{6}$ Esto es importante pese a su infradiagnóstico, debido a las implicaciones clínicas en cuanto a los errores quirúrgicos que puede inducir, falta de correlación clínica de diagnósticos como puede ser la asociación a la lumbalgia, el síndrome de Bertolotti, inestabilidad, degeneración articular, compresión nerviosa o hipertrofia de tejidos, ${ }^{7}$ ya que las alteraciones generadas por la vértebra de transición, como el dolor de espalda secundaria, incluyen alteraciones como cambios en el canal espinal, patología de la transición, espondilosis, degeneración contralateral anormal y compromiso extraforaminal. ${ }^{8}$

\section{Diagnóstico}

De manera original, se realizó mediante el uso de la radiografía, empleando la toma lateral y las radiografías de Ferguson. Otras características morfológicas de la vértebra de transición incluyen la cuadratura del segmento sacro superior en el proceso de lumbarización y acuñamiento del segmento lumbar inferior en la sacralización. ${ }^{8}$
Dichos cambios morfológicos representan o involucran un desplazamiento craneal o caudal de la columna, resultando en un menor número de segmentos de movimiento en la columna; por lo que se ha descrito que las vértebras de transición tienen una apariencia cuadrada con una relación AP de la vertebral superior e inferior $\leq 1.37 .^{9}$

En 1984, mediante el diagnóstico radiográfico, se realizó una clasificación de la anormalidad de la vértebra de transición, la cual se estableció de la siguiente forma (Figura 1):

Tipo I. Procesos de transición displásicos con una longitud de al menos 19 mm en dirección cráneo caudada, la cual tiene una subdivisión:

Tipo la: unilateral.

Tipo Ib: bilateral.

Tipo II. Proceso de lumbarización o sacralización incompleto con un proceso transversal agrandado, que tiene una articulación artrodial entre ella y el sacro. Tipo Ila: unilateral.

Tipo Ilb: bilateral.

Tipo III. Lumbarización o sacralización completa con una fusión ósea de procesos transversos al sacro.

Tipo Illa: unilateral.

Tipo IIlb: bilateral.

Tipo IV. Involucra un proceso de transición unilateral de tipo II con alteraciones contralaterales tipo III.

Siendo que esta clasificación presenta o caracteriza la relación entre los segmentos transicionales y el segmento superior o inferior de la vértebra, pero como inconveniente no provee información relevante sobre los segmentos que están involucrados en la alteración. ${ }^{4}$

También se posee en la actualidad una clasificación realizada para la identificación de las anormalidades en los discos intervertebrales, la cual es conocida como clasificación de O'Driscoll que incluye o considera los siguientes cuatro tipos: ${ }^{10}$

Tipo 1: no hay material discal entre S1 y S2, observada como una línea hipotensa en los estudios de resonancia magnética o tomografía computada.

Tipo 2: pequeños discos entre $\mathrm{S} 1$ y el sacro, que no se extiende en todo el diámetro sacral anteroposterior.

Tipo 3: disco bien formado entre S1 y el sacro, que se extiende a todo el diámetro sacral AP.

Tipo 4: disco bien formado entre S1 y el sacro que se extiende a todo el diámetro sacral AP, con cuadratura del borde sacro superior.

En la actualidad se ha señalado que existe disponible y validado el uso de la tomografía computada y la resonancia magnética para el diagnóstico y descripción de la vértebra de transición. El uso de estos estudios de imagen 

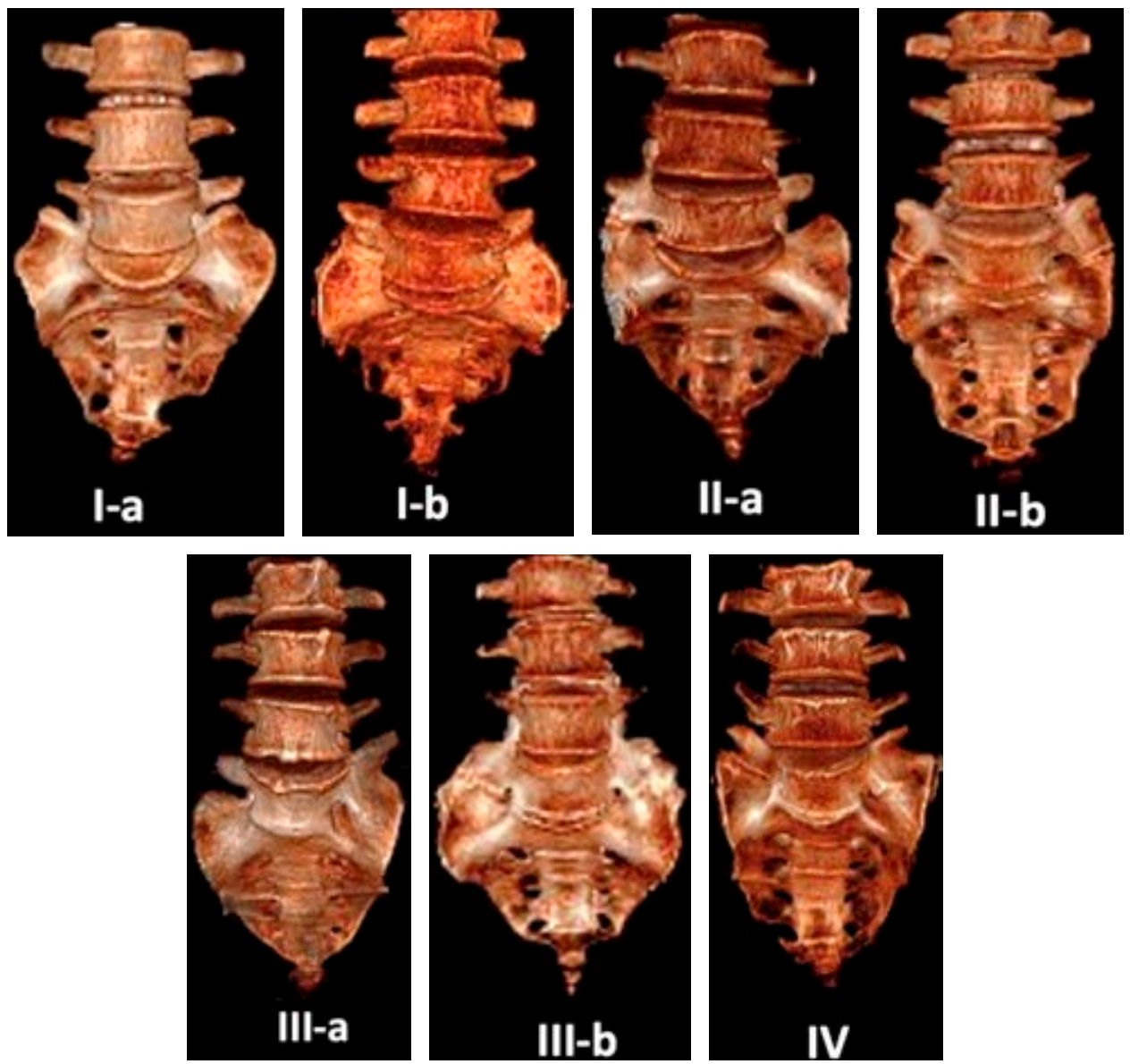

Figura 1:

Clasificación de la anormalidad de la vértebra de transición.

nos permite identificar cambios mecánicos asociados con la distribución del peso y las modificaciones articulares de las vértebras, ${ }^{11,12}$ empleando los mismos criterios diagnósticos para vértebras y discos. ${ }^{13}$

Los estudios de imagen más actuales mantienen una correlación directa con los resultados sobre vértebra de transición identificados mediante la radiografía. ${ }^{14}$ Se ha demostrado que el uso de la tomografía computada en reconstrucción coronal es más útil para la detección de las conexiones óseas y de los procesos transversales, en especial de L5 y la ala sacra. ${ }^{15,16}$

La vértebra de transición es una anormalidad que, si bien no cabe en la categoría de ser una rareza, su diagnóstico o identificación sí se da de manera incidental, por lo que muchas veces se presenta de manera infradiagnosticada, lo cual es observable por el amplio rango de incidencia que se ha señalado en la literatura.

No se cuenta con literatura de la incidencia y características propias en una población mexicana; esta información permitirá identificar características propias de la vértebra de transición que permitirá mejorar el diagnóstico, o bien, optimizar y mejorar su conocimiento.
El sistema óseo puede presentar distintos tipos de anomalías que pueden ser observadas a simple instancia, de diagnóstico fácil o de hallazgo incidental; dentro de estas últimas se encuentra la vértebra de transición, cuya incidencia alcanza hasta un 23\%, pero su diagnóstico es casual o incidental.

Aunque no significa que la vértebra de transición no produzca un conjunto de alteraciones clínicas o sintomatología que genere desde dolor hasta inestabilidad. Sin embargo, debido a su hallazgo casual, muchas veces está infradiagnosticado y los conocimientos epidemiológicos de esta alteración no son claros.

Objetivo. Determinar la incidencia de la anormalidad de la vértebra de transición por tomografía computada en la población mexicana.

\section{MATERIAL Y MÉTODOS}

Estudio observacional, analítico, retrospectivo y transversal. Se llevó a cabo en la población mexicana atendida en el Servicio de Radiología e Imagen del Hospital Mocel Angeles entre enero de 2017 y septiembre de 2020, donde se re- 
visaron todos los casos de individuos sometidos a estudios de tomografía computada sin importar motivo de ingreso al servicio para el estudio de la vértebra de transición, excluyendo a pacientes de origen extranjero para delimitar una muestra plenamente mexicana corroborando su lugar de nacimiento. En este estudio se tuvo un total de 860 pacientes tomando en cuenta los criterios de inclusión.

Criterios de inclusión: pacientes de origen mexicano, mayores a 18 años, atendidos en el Servicio de Radiología e Imagen del Hospital Angeles Mocel, durante el periodo establecido y que contaran con tomografía computada por cualquier motivo de ingreso al servicio.

Criterios de exclusión: se eliminó a aquéllos menores de 18 años, que fueran extranjeros, embarazadas o que tuvieran un estudio de imagen diferente a la tomografía computada.

Criterios de eliminación: expedientes o instrumentos incompletos.

Variables dependientes, independientes y de confusión: vértebra de transición, sacralización, lumbarización, género, edad, tipo de lesión de vértebra de transición, pseudodisco y morfología del disco sacral.

Variables por estudio de imagen: todas las muestras del presente estudio se sometieron de acuerdo con el comité de ética, que fue aceptado y se procedió a revisar los registros del área de tomografía computada para indagar los casos que hayan sido sometidos a evaluación en busca de cambios morfológicos entre enero de 2017 y septiembre de 2020. Se utilizó el equipo General Electric Light Speed de 16 cortes con filtros de alta resolución con reconstrucciones en ventana ósea y de tejidos blandos, así como con reconstrucción 3D. De los casos que cumplieron con los criterios de selección, se obtuvieron de los expedientes clínicos y los estudios de imagen las variables antes señaladas.

El análisis estadístico se llevó a cabo empleando el programa IBM SPSS v26 (USA). Los resultados fueron expresados en tablas y gráficas según corresponda. Los análisis de variables cualitativos se realizaron con frecuencias y porcentajes, mientras que las variables cuantitativas fueron analizadas mediante medidas de tendencia central. Se analizó la distribución de la muestra con base en la edad con la prueba de Kolmogórov-Smirnov. Los análisis comparativos de variables cuantitativas se realizaron con pruebas paramétricas o no paramétricas según corresponda entre los grupos con y sin vértebra de transición. Se considerará prueba estadísticamente significativa cuando $\mathrm{p} \leq 0.05$.

\section{RESULTADOS}

Entre enero de 2017 y septiembre de 2020 se estudiaron en el Departamento de Radiología e Imagen a 860 pacientes que cumplieron con los criterios de selección señalados en el presente protocolo. De los cuales 52.8\% fueron mujeres $(n=454)$ y $47.2 \%(n=406)$ eran hombres.

La edad media de la población estudiada fue de 51.99 \pm 20.54 años, con una distribución según la prueba de Kolmogórov-Smirnov no normal o no paramétrica $(p=$ 0.03). De los 860 casos incluidos en el estudio, 17.4\% (n $=150$ ) presentó diagnóstico de vértebra de transición por estudio de imagen de tomografía; teniendo que 54 casos (6.27\% de la población o 36\% de los casos con vértebra de transición) presentaron procesos de lumbarización, mientras que 96 casos presentaron procesos de sacralización (11.2\% de la población o 64\% de los casos de vértebra de transición). La incidencia de tipos de vértebra de transición se muestra en la Tabla 1.

En cuanto a la revisión de alteraciones de disco intervertebral, se encontraron 146 casos con alteraciones (16.97\%), de los cuales el tipo IV fue el más común (10.7\%) seguido de 32 casos del tipo III (3.7\%), 16 del tipo II (1.8\%) y el menos común fue el tipo I con seis casos (0.7\%).

De los 430 casos incluidos en el estudio, se encontraron 34 (4\%) con escoliosis, de los cuales 20 (2.3\%) presentaron escoliosis a la izquierda y el resto a la derecha. Por último, en relación a las vértebras involucradas o afectadas, en 92 expedientes revisados la afectada fue L5 y en 29 la S1. No se encontró asociación significativa entre el diagnóstico de vértebra de transición con la edad o el sexo.

\section{DISCUSIÓN}

Acorde a nuestro estudio, se puede observar que la mayoría de los casos fueron mujeres, en los cuales fue realizada una búsqueda dirigida en la zona lumbar, lo cual concuerda con lo reportado en la investigación de las lumbalgias en cuanto a la prevalencia de género en mujeres. ${ }^{17-19}$

Tabla 1: Tipos de vértebra de transición.

\begin{tabular}{cccc} 
Tipo & $\mathrm{n}$ & $\begin{array}{c}\text { Población } \\
\text { total (\%) }\end{array}$ & $\begin{array}{c}\text { Población con vértebra } \\
\text { de transición (\%) }\end{array}$ \\
\hline I & & & \\
Ia & 10 & 1.2 & 6.66 \\
Ib & 48 & 5.6 & 32.00 \\
II & & & \\
IIla & 14 & 1.6 & 9.33 \\
IIb & 34 & 4.0 & 5.33 \\
III & & & 2.66 \\
IIIa & 4 & 0.5 & 16.00 \\
IIIb & 24 & 2.8 & 8.00 \\
IV & 12 & 1.4 &
\end{tabular}


Empero la diferencia se presentó en relación con la edad de la población estudiada, ya que nuestro valor de media y desviación estándar coloca la edad por encima de la media de edad de la lumbalgia. ${ }^{19,20}$

En cuanto a la incidencia de la vértebra de transición se encontró que en nuestra población de estudio se encontró del rango de incidencia que se ha descrito para esta enfermedad que oscila entre 4 y $30 \% .^{21,22}$

En cuanto a la prevalencia por sexo o género, no se encontró asociación alguna que corresponda con lo que se ha descrito hasta la actualidad, ya que, si bien en algunos casos parece reportarse un predominio de género o edad, ésta no se repite o se demuestra de manera estadísticamente significativa. ${ }^{22}$ Tampoco se encontró alguna asociación entre la edad, sexo con la lateralidad o tipo de vértebra de transición. ${ }^{21}$

\section{CONCLUSIÓN}

La incidencia de vértebra de transición en la población mexicana presenta una incidencia de $17.4 \%$, similar a lo reportado en otras latitudes, con características similares a lo señalado para la vértebra de transición.

Sin embargo, debido a su hallazgo incidental muchas veces está infradiagnosticada y los conocimientos epidemiológicos no son claros. Todo lo anterior conlleva que con esta alteración se puede asociar lumbalgia, síndrome de Bertolotti, inestabilidad, degeneración articular, compresión nerviosa o hipertrofia de tejidos.

\section{AGRADECIMIENTOS}

Se agradece de manera particular por sus contribuciones al Servicio de Radiología e Imagen del Hospital Angeles Mocel y al Dr. José Luis Ramírez Arias por su orientación en la realización del artículo.

\section{REFERENCIAS}

1. Murlimanju BV, Prabhu LV, Pai MM, Ganeshkumar C, Sarvepalli A. Lumbosacral transitional vertebrae: a case report and clinical implications. Int J Morphol. 2011; 29 (4): 1123-1125.

2. Bron JL, van Royen BJ, Wuisman PI. The clinical significance of lumbosacral transitional anomalies. Acta Orthop Belg. 2007; 73 (6): 687-695.

3. Tins BJ, Balain B. Incidence of numerical variants and transitional lumbosacral vertebrae on whole-spine MRI. Insights Imaging. 2016; 7 (2): 199-203.

4. Konin GP, Walz DM. Lumbosacral transitional vertebrae: classification, imaging findings, and clinical relevance. AJNR Am J Neuroradiol. 2010; 31 (10): 1778-1786.

5. Carapuco M, Nóvoa A, Bobola N, Mallo M. Hox genes specify vertebral types in the presomitic mesoderm. Genes Dev. 2005; 19 (18): 2116-2121.
6. Noguerol TM, Barbero JPM, Broncano J. i L5 o S1? Valoración de las anomalías de transición lumbosacra mediante RM. Radiología. 2014; 56 (Espec Cong): 816.

7. Taskaynatan MA, Izci Y, Ozgul A, Hazneci B, Dursun H, Kalyon TA. Clinical significance of congenital lumbosacral malformations in young male population with prolonged low back pain. Spine (Phila Pa 1976). 2005; 30 (8): E210-E213.

8. Castellvi AE, Goldstein LA, Chan DP. Lumbosacral transitional vertebrae and their relationship with lumbar extradural defects. Spine (Phila Pa 1976). 1984; 9 (5): 493-495.

9. Dai L. Lumbosacral transitional vertebrae and low back pain. Bull Hosp Jt Dis. 1999; 58 (4): 191-193.

10. O'Driscoll CM, Irwin A, Saifuddin A. Variations in morphology of the lumbosacral junction on sagittal MRI: correlation with plain radiography. Skeletal Radiol. 1996; 25 (3): 225-230.

11. Mahato NK. Facet dimensions, orientation, and symmetry at L5-S1 junction in lumbosacral transitional States. Spine (Phila Pa 1976). 2011; 36 (9): E569-E573.

12. Pekindil G, Sarikaya A, Pekindil Y, Gültekin A, Kokino S. Lumbosacral transitional vertebral articulation: evaluation by planar and SPECT bone scintigraphy. Nucl Med Commun. 2004; 25 (1): 29-37.

13. Farshad-Amacker NA, Herzog RJ, Hughes AP, Aichmair A, Farshad $M$. Associations between lumbosacral transitional anatomy types and degeneration at the transitional and adjacent segments. Spine J. 2015; 15 (6): 1210-1216.

14. Aihara T, Takahashi K, Ogasawara A, Itadera E, Ono Y, Moriya $H$. Intervertebral disc degeneration associated with lumbosacral transitional vertebrae: a clinical and anatomical study. J Bone Joint Surg Br. 2005; 87 (5): 687-691.

15. Lee CS, Ha JK, Kim DG, Hwang CJ, Lee DH, Cho JH. The clinical importance of lumbosacral transitional vertebra in patients with adolescent idiopathic scoliosis. Spine (Phila Pa 1976). 2015; 40 (17): E964-E970.

16. Iwasaki M, Akiyama M, Koyanagi I, Niiya Y, Ihara T, Houkin K. Double crush of L5 spinal nerve root due to L4/5 lateral recess stenosis and bony spur formation of lumbosacral transitional vertebra pseudoarticulation: a case report and review. NMC Case Rep J. 2017; 4 (4): 121-125.

17. Matson DM, Maccormick LM, Sembrano JN, Polly DW. Sacral Dysmorphism and Lumbosacral Transitional Vertebrae (LSTV) Review. Int J Spine Surg. 2020; 14 (Suppl. 1): 14-19. doi: 10.14444/6075.

18. Sabogal IDRE, Arrieta MLJ, Arrieta LR, Flórez MSR. Casos de lumbalgia en trabajadores de facturación central. Clínica San Juan de Dios Cartagena-2011. Biociencias. 2014; 9 (2): 77-86.

19. Soto-Padilla M, Espinosa-Mendoza RL, Sandoval-García JP, GómezGarcía F. Frecuencia de lumbalgia y su tratamiento en un hospital privado de la Ciudad de México. Acta Ortop Mex. 2015; 29 (1): 40-45.

20. Escalona E. Factores de riesgos ocupacionales y consideraciones de género en los estudios epidemiológicos de las lumbalgias. Salud de los Trabajadores. 2000; 8 (1): 51-76.

21. Hsieh CY, Vanderford JD, Moreau SR, Prong T. Lumbosacral transitional segments: classification, prevalence, and effect on disk height. J Manipulative Physiol Ther. 2000; 23 (7): 483-489.

22. Mahato NK. Relationship of sacral articular surfaces and gender with occurrence of lumbosacral transitional vertebrae. Spine J. 2011; 11 (10): 961-965.

Conflicto de intereses y financiamiento: Ninguno. 